\title{
Los primeros principios como intelección del ser en cuanto primero
}

First Principles as Intellection of Being as First

Alfredo Rodríguez Sedano

Universidad de Navarra

arsedano@unav.es

\author{
Ana Costa Paris \\ Universidad de Navarra \\ acosta@unav.es
}

Resumen: El objetivo que se persigue en este trabajo es insinuar cómo se puede acceder, a través del principio de identidad, al conocimiento natural de Dios que se alcanza en la primera dimensión del abandono del límite mental. Comenzaremos por ver qué entiende Polo por límite mental y cómo es posible abandonarlo. La razón que aduce Polo para que el abandono del límite mental pueda darse es el conocimiento habitual, superior a la operación. Al tratarse de la primera dimensión el estudio se centra en los Primeros Principios. Se verá qué significa conocer habitualmente y qué es lo peculiar del hábito de los primeros principios. Concluiremos en ver cuál es el conocimiento de Dios que se alcanza en la primera dimensión del abandono del límite mental.

Palabras clave: Conocimiento, abandono límite, hábitos, Dios, primeros principios.
Abstract: The objective of this study id to suggest how one access, through the principle of identity, the natural knowledge that in God which is reached in the first dimension of the abandonment of mental limit. We will start by looking at what Polo understands by mental limit and how it is possible to abandon it. The reason that Polo gives for the possibility of abandonment of mental limit is habitual knowledge, which is superior to the operation. Dealing with the first dimension, this study will focus on the habit of the first principles. We will also look at what it means to know habitually and what is proper to the habit of the first principles. Finally, we will study the knowledge of God that can be reached in the first dimension of the abandonment of mental limit.

Keywords: Knowledge, Abandonment of Mental Limit, Habits, God, First Principles. 


\section{LA LIMITACión DEL CONOCIMIENTO OBJETIVO}

A unque de suyo es conocido, la propuesta del límite mental en Polo tiene su origen histórico cuando "en la primavera de 1950, Leonardo Polo cae en la cuenta del límite mental". La indagación es narrada por el mismo Polo: "eso se me ocurrió de repente, y punto. Estaba pensando acerca del pensar y el ser, y cómo tenía que ver el ser con el pensar; entonces me di cuenta de que al ser no podíamos llegar mientras no se abandonara la suposición del objeto, porque la suposición hace que el objeto sea limitado y un conocimiento limitado no puede ser un conocimiento del ser si éste se toma en sentido trascendental"'. Puede afirmarse, de acuerdo a esta intencionalidad y posterior desarrollo, que toda la filosofía de Leonardo Polo es una consideración de la operación mental del hombre 3 .

De modo preciso lo que Polo entiende por límite mental es que el conocimiento intencional es capaz de pensar una infinitud de objetos, cuya característica común es que coinciden en ser objeto. Desde esa perspectiva no cabe hablar de ganancia del conocimiento. Pues en eso es en lo que consiste el límite del pensamiento. El límite del pensamiento es la objetivación. "Cabe pensar infinitud de objetos, pero todos ellos coinciden en serlo, y en dicha medida no se ha ganado nada; a eso llamo el límite del pensamiento" ${ }^{4}$. Y en otra ocasión señala: "el límite mental es justamente el conocimiento objetivo intelectual" " Y en otro momento, "la constancia de la presencia es el límite mental"6.

Así, para Polo la detectación del límite mental establece que éste es el objeto pensado, en cuanto supuesto o pensado. La limitación del pensamiento "se cifra, en última instancia, en la detención cognoscitiva que introduce cualquier objeto pensado -que también recibe el nombre de suposición-. En efecto, en tanto que ya he pensado un objeto, sólo pienso en lo pensado, por lo que he dejado de pensar en más objetos y he dejado de conocer mejor la realidad -he

\footnotetext{
${ }^{1}$ M. J. FRANQUET, “Trayectoria intelectual de Leonardo Polo”, en Anuario Filosófico, XXIX/2 (1996), 305.

2 Ibidem.

3 Así lo sostiene J. GARCÍA GONZÁLEZ, "El abandono del límite mental”, en Anuario Filosófico, XXV/1 (1992), 52.

${ }^{4}$ L. POLO, Curso de teoría del conocimiento III, 372.

${ }^{5}$ L. POLO, El conocimiento habitual de los primeros principios, Cuadernos de Anuario Filosófico, Servicio de Publicaciones de la Universidad de Navarra, 10, Pamplona, 1993, 7.

${ }^{6}$ L. POLO, Curso de teoría del conocimiento II, 153.
} 
supuesto la realidad- (...). En cierto sentido, al pensar se deja de pensar; pero también al revés, gracias a que dejo de pensar, al pensar lo pensado, puedo seguir pensando más"’.

Precisamente porque los objetos coinciden en ser pensados, en esa misma medida no hemos ganado nada. La actualidad no conlleva ganancia respecto a lo pensado, ya que no aumenta su carácter de pensado8.

A esa actualidad de lo pensado que no es posible ir más allá de lo pensado es a lo que Polo denomina límite mental. Si el límite mental es realmente un descubrimiento está apuntando de lleno a la pregunta por el ser. Lo que conlleva un verdadero descubrimiento, con la connotación de hallazgo que el término sugiere. "Si se puede destacar a Polo dentro de la historia de la filosofía es justamente porque es el primer filósofo que adopta como método el abandono del límite mental; es el primer filósofo en establecer metódicamente cuáles son las condiciones para detectar y abandonar de un modo fructífero la limitación que introduce el objeto pensado".

Detenerse a pensar no comporta, por consiguiente, un aumento del conocimiento, puesto que el objeto $y a$ ha sido pensado. El carácter de $y a$ se refiere a la situación de lo pensado y de este modo puede decirse que lo pensado no piensa. Hay una neta distinción entre acto y actualidad. Para explicar esa distinción Polo dice que "suelo emplear para el caso la fórmula el yo pensado no piensa, que vale según la constancia del estatuto de los objetos pensados (presencia mental). Ningún objeto piensa: puedo pensar que pienso, pero no puedo de ninguna manera comunicar mi carácter pensante a lo que pienso" ${ }^{10}$.

Según lo dicho puede entenderse que el objeto pensado es una versión de la realidad, pero no es real. "El objeto pensado no es trascendental, sino intencional". La equiparación entre algo y objeto pensado se establece en el Curso de teoría del conocimiento II ${ }^{11}$. Por tanto, "sólo cabe decir que un que es distinto de otro en tanto que pensado" ${ }^{12}$.

Lo real es el acto, mientras que el objeto es algo así como detenido, a quien conviene el nombre de actualidad. Hay, pues, una clara distinción entre

\footnotetext{
7 S. PIÁ TARAZONA, El hombre como ser dual. Estudio de las dualidades radicales según la Antropología trascendental de Leonardo Polo, Eunsa, Pamplona, 2001, 16-17.

${ }^{8}$ Cfr. L. POLO, Curso de teoría, I, 57.

9 S. PIÁ TARAZONA, El hombre como ser dual, 16.

${ }^{10}$ L. POLO, Curso de teoría del conocimiento III, 306.

11 L. POLO, Curso de teoría del conocimiento II, 79-106.

12 L. POLO, Antropología I, 70, nota 67.
} 
acto -lo real- y la actualidad -el objeto pensado-. No reducir el acto a actualidad es la clave que nos presenta Polo para ir más allá del límite mental, de la presencia mental, de la actualidad, del ya de lo pensado ${ }^{13}$.

Recordemos que en el conocimiento objetivo se da la actualidad, mientras que en la concentración atencional se mantiene la concentración en el acto no reduciéndolo a actualidad. Tomás de Aquino ya lo había visto anteriormente. Parafraseando una conocida tesis suya, se puede decir que "lo primero que acaece al intelecto es la realidad" ${ }^{14}$.

Matiza Polo respecto al conocimiento objetivo que "empleo la palabra objeto en un sentido preciso. Muchas veces se habla de objeto como cosa, pero objeto significa estrictamente lo conocido en tanto que conocido, cuando se conoce operativamente" ${ }^{\prime 15}$. Y esto es lo propio de las operaciones cognoscitivas. Por consiguiente si los objetos se conocen ejerciendo operaciones, "la limitación del conocimiento objetivo debe estar estrechamente unida con la operación" ${ }^{\prime 16}$.

La limitación del conocimiento objetivo es patente en la medida en que el pensar no agota lo real. En este sentido es certera la frase que se atribuye a Tomás de Aquino: "hay más realidad en una mosca que en la mente de todos los filósofos". Efectivamente en la mente humana no hay realidad física alguna sino ideas. Por otra, siempre hay más mosca real por pensar que lo que se ha pensado de la mosca ${ }^{17}$.

Las operaciones, por consiguiente poseen objetos. La cuestión está en que si no hubiese otro tipo de conocimiento, el límite no se podría abandonar. Se precisa un tipo de actos cognoscitivos superiores. Y esos actos son los há-

13 El estudio del abandono del límite mental lo lleva a cabo Polo en los siguientes textos. Del abandono del límite mental -detectarlo y abandonarlo- se ocupa El acceso al ser. Después de decir qué significa abandonar el límite mental, o sea, de exponer o establecer la metodología, escribe $E l$ ser I, que trata de la existencia extramental. El ser I es la formulación de la temática metafísica desde el abandono del límite mental. El tomo IV del Curso de teoría del conocimiento, se ocupa de la esencia extramental. En ese libro se expone de qué manera se abandona el límite mental para advertir los primeros principios (el ser de que trata la metafísica) y las causas predicamentales (es decir, la esencia extramental en cuanto distinta realmente del ser extramental). La Antropología I, se ocupa de la coexistencia humana -el ser humano-. Finalmente, la Antropología II, se ocupará de la esencia humana.

${ }^{14}$ TOMÁS DE AQUINO, Summa Theologiae I-II, q. 55, a. 4 ad 1; De Potentia, q. 9 a7 ad 15.

15 L. POLO, Nominalismo, idealismo y realismo, 190.

${ }^{16}$ Ibidem, 193.

17 Esto ha sido puesto de relieve entre otros por J. PIEPER, "El filosofar y el lenguaje", en Anuario Filosófico, XXI/1 (1988), 74. M. D. CHENU, Introduction al'etude de Saint Thomas d'Aquin, París-Montreal, 1959, 102. 
bitos. "Si no tuviésemos más actos intelectuales que las operaciones, no podríamos abandonar el límite, puesto que el límite es el objeto y, correlativamente, la operación. Conocer operativamente es conocer objetivamente y, por tanto, limitadamente. Para que el abandono del límite no pase de ser un anhelo irrealizable, tiene que existir un tipo de actos cognoscitivos superiores a las operaciones, que son los hábitos intelectuales"18.

Y ésta es la tarea propia de los hábitos intelectuales en cuanto que "nos permiten advertir la realidad en su propio ser, en su propia actividad, y no perder la atención respecto de tal actividad" ${ }^{19}$. Atención que se llegaría a perder si se introduce el conocimiento objetivo, ya que entonces, reduciríamos la actividad a algo pensado ${ }^{20}$.

Pero, téngase en cuenta, que todo lo pensado, en tanto que es algo pensado, se piensa como identidad estática; es decir, se piensa como algo ya constituido y determinado, como algo idéntico a sí mismo. De este modo, el conocer consistiría en hacer conexiones entre objetos ya pensados. Ahora bien, en las conexiones mentales no se profundiza "en orden a ese $y a$, porque también lo que se añade es ya pensado"'1.

Merece la pena hacer una breve alusión al hecho de que ésta no es una idea que Polo tenga y sostenga alejada de la Tradición -con mayúscula- filosófica, otra cuestión es que la matice ${ }^{22}$. Tomás de Aquino en De Veritate afirma que aquel conocimiento que es sumo en el hombre no es un haber, no es posesión, sólo lo es en Dios mismo, como ya observó Aristóteles ${ }^{23}$. Con ello queda establecido de golpe que el conocimiento intelectual es creado, que le corresponde una actualidad virtualmente infinita y que, por ello, se conoce o reconoce como creado (participatur). Y, a continuación, afirmará que éste es un conocer natural y habitual: qui dicitur intellectus principiorum, llamado inteligencia de los principios.

${ }^{18}$ L. POLO, Nominalismo, idealismo y realismo, 193.

19 S. PIÁ TARAZONA, "De la criatura a Dios. La demostración de la existencia de Dios en la primera dimensión del abandono del límite mental", en Anuario Filosófico, XXIX/2 (1996), 930.

20 "Pensar es siempre pensar algo", I. FALGUERAS, "Los planteamientos radicales de la filosofía de Leonardo Polo", en Anuario Filosófico, XXV/1 (1992), 77.

${ }^{21}$ H. ESQUER, Actualidad y acto, 147.

${ }^{22}$ Cuando nos refiramos a la Tradición filosófica, salvo que se diga algo distinto, nos estamos refiriendo a Tomás de Aquino y Aristóteles, que forman parte del acervo filosófico de Leonardo Polo.

23 "Id autem quod sic participatur, non habetur ut possessio, id est sicut aliquid perfecte subiacens potentiae habentis illud; sicut dicitur in I Metaphysicorum, quod cognitio Dei divina est, non humana possessio"; TOMÁS DE AQUINO, De Veritate, q. 15, a. 1, c. 
En este sentido para Tomás de Aquino "hay un pensar en acto el ser como principio, y remite al Ser como identidad. Y ¿por qué 'remite'? Porque ningún ente es evidente por sí mismo y en atención sólo a él mismo; si la evidencia se define como una identidad original que se analiza como sujeto y predicado, entonces la verdad original antecede a la predicación" ${ }^{\prime 24}$.

De acuerdo con lo señalado, para Tomás de Aquino la verdad original es el ser, luego no es la criatura ${ }^{25}$. Lo que equivale a decir que si la criatura es y se entiende por el ser, no se entendería que fuera absoluta. O que el ser se reserva siempre, como principio primero, la alteridad. Y esa alteridad es lo que se mantiene más allá del límite mental. Luego conocer la alteridad es conocer más intensamente.

En cambio, como ya hemos señalado, Polo llama "límite" a las operaciones del intelecto, en cuanto operaciones inmanentes. La señal de su limitación está en su pluralidad y en su intencionalidad. Todo conocimiento intencional es parcial, luego "en términos objetivos no tenemos un conocimiento absoluto, y, todavía menos, un conocimiento absoluto del Absoluto"26.

Hemos esbozado la posibilidad de detectar el límite y abandonarlo, con la finalidad de ver que se puede llegar a algo más que el conocimiento objetivo. Sin embargo, este intento ha sido frecuente en la historia de la Filosofía. No obstante este abandono no ha de asimilarse a los intentos modernos que se han llevado a cabo. Así lo señala expresamente Polo: "abandonar el conocimiento objetivo no es tirar la escalera, es decir, apelar -al modo de Espinosaa una intuición, ni desembocar en una contemplación especulativa, aunque sea tan pura como la de Escoto. Tampoco es la superación nietzscheana, pues sería inútil destruir el objeto para llegar a una construcción nueva, hecha recombinando trozos de la anterior. Asimismo, es diferente del término del proceso dialéctico de Hegel, ya que a ningún objeto puede alcanzar la condición de sujeto; y de la idea de abarcador de Jaspers"27.

${ }^{24}$ S. FERNÁNDEZ-BURILLO, “'Intellectus principiorum': de Tomás de Aquino a Leonardo Polo", en Anuario Filosófico, XXIX/1 (1996), 517.

25 "Hoc autem quod est esse, in nullius creaturae ratione perfecte includitur; cuiuslibet enim creaturae esse est aliud ab eius quidditate [distinción real, criatura]: unde non potest dici de aliqua creatura quod eam esse sit per se notum et secundum se [causalidad]. Sed in Deo esse suum includitur in eius quidditatis ratione, quia in Deo idem est quid esse et esse [...]; , et idem est an est et quid est [identidad], [...]; et ideo per se et secundum se est notum"; TOMÁS DE AQUINO, De Veritate, q. 10, a. 12, c.

${ }^{26}$ L. POLO, El conocimiento habitual de los primeros principios, 8.

27 L. POLO, Antropología I, 119. 
La clave para resolver la cuestión de lo transobjetivo radica en actos intelectuales superiores a las operaciones inmanentes. Esos actos intelectuales son los hábitos que permiten no sólo detectar el límite mental sino llevar a cabo el abandono de ese límite. Por esta razón, Polo señala que "es incorrecto sostener que el hombre sólo conoce objetivamente; pero para llegar más lejos no hay que apelar a la voluntad, ni a la intuición emocional, ni a actitudes que lindan con la mística o con la teología negativa”28.

\section{Conocimiento habitual}

Y la razón que aduce Polo para que el abandono del límite mental pueda darse parece clara: "para que el abandono del límite no pase de ser un anhelo irrealizable tiene que existir un tipo de actos cognoscitivos superiores a las operaciones, que son los hábitos intelectuales. Dentro de este tipo de actos, el conocimiento de lo principial, del protón, es el conocimiento más profundo, y no puede decirse que sea aspectual, porque no es objetivo. Con todo, éste no es el hábito superior, porque por encima de él está el hábito de la sabiduría, y, por encima de éste, otros que son sobrenaturales...”29.

El planteamiento de la primera dimensión del abandono del límite mental queda planteado. "Sin conocimiento habitual, lo que llamo abandono del límite mental no se entiende, y en teoría del conocimiento no se puede justificar. Repito. Si el conocimiento limitado es el conocimiento operativo-objetivo, sólo si además de las operaciones existe otro tipo de actos, que son los hábitos, podemos abandonar el límite: si no, la limitación de nuestro conocimiento sería insuperable" ${ }^{30}$.

La novedad que radica en el modo de entender los hábitos intelectuales por parte de Polo respecto a los clásicos, es claramente entender que los hábitos son actos cognoscitivos superiores a lo que se entiende por conocimiento operativo. Y se advierte de la novedad por cuanto que el tratamiento usual de los hábitos intelectuales no incide en su carácter cognoscitivo. "La doctrina normal -señala Polo- es que los hábitos intelectuales se asimilan a la facultad y son perfectivos de ella. Para avanzar en la consideración de su valor estrictamente cognoscitivo, hay que atender a su relación con el intelecto agente" ${ }^{{ }^{31}}$.

\footnotetext{
28 Ibidem, 121.

29 Ibidem, 9.

30 L. POLO, Nominalismo, idealismo y realismo, 194.

31 Ibidem, 195.
} 
La diferencia clara que se encuentra en el planteamiento de Polo respecto a la propuesta del Aquinate radica en que para Polo el hábito intelectual no es resultado de otra operación (enérgeia), ni de una potencia o acto constitutivo (entelékheia). Éste es el punto de mayor diferencia con Tomás de Aquino: el hábito no se asimila a la potencia, sino a la luz del intelecto agente, que acompaña al mismo esse hominis: "al hábito intelectual hay que buscarle una línea de entrada por el actus essendi (...) El conocimiento habitual es una iluminación debida al intelecto agente (el cual es asimilable al actus essendi) ${ }^{32}$ ".

$\mathrm{El}$ intelecto agente ya aparece en la tradición filosófica, aunque con matices diversos. "Los modos de conocer que Polo advierte en la cima del conocimiento natural humano están esbozados en la tradición filosófica aristotélicotomista. El superior de ellos es el denominado -desde Aristóteles-intelecto agente, tal vez el descubrimiento gnoseológico más relevante de la historia de la filosofía, el más comentado a lo largo del aristotelismo medieval y renacentista" ${ }^{\prime 3}$.

Efectivamente, para Polo, el intelecto agente es equivalente al actus essendi hominis ${ }^{34}$, tesis que no estableció Tomás de Aquino por considerarlo como una "potencia" ${ }^{35}$. Junto al intelecto agente, otros niveles cognoscitivos que son superiores a la razón, e inferiores al entendimiento en acto, son los hábitos de la sabiduría y de los primeros principios, descubiertos por Aristóteles, y el hábito de la sindéresis atribuido a san Jerónimo, quien la denominó "la chispa de la conciencia" ${ }^{36}$.

Estos hábitos son considerados por Polo como instrumentos del entendimiento agente para liberarle de tareas inferiores. El tema del intelecto agente es superior al del hábito.

La vigencia de estos hábitos no es tampoco algo nuevo. La tradición filosófica medieval consideraba innatos a los primeros principios y a la sindéresis. El hábito de los primeros principios está "inserto en nosotros" ${ }^{37}$ y "se tiene por

${ }^{32}$ L. POLO, El conocimiento habitual de los primeros principios, 18.

33 J. F. SELLÉS, "Los niveles cognoscitivos superiores de la persona humana", en Studia Poliana, 10 (2008), 53.

${ }^{34}$ L. POLO, Nominalismo, idealismo y realismo, 187.

35 Cfr. J. F. SELLÉS, "El entendimiento agente según Tomás de Aquino", en Revista Española de Filosofía Medieval, 9 (2002), 105-124.

36 "Scintilla conscienciae in Cain quoque pectore, postquam eiectus est de Paradiso, non extinguitur, et qua victi voluptatibus, vel furore, ipsaque interdum rationis decepti similitudine, nos peccare sentimus", Glossa ordin. Ezechiel, I, c. 1 (PL 25, 22 A-B).

37 "Habitam illum primorum principiorum quorum cognitio nata aliter est ínsita nobis secundum quod in omnibus iudiciis dirigimur". In II Sent., d. 24, q. 1, a. 1, ad 3; "sunt nobis indita". TOMÁS DE AQUINO, Summa Theologica, I, q. 79, a. 12, co; "prima autem rationis principia 
naturaleza" "38. "la sindéresis... es en cierto modo innato a nuestra mente" ${ }^{39}$. Se sostenía, en cambio, que el de sabiduría es adquirido ${ }^{40}$. No obstante, la tradición filosófica medieval también admitía la existencia de un hábito originario que permite el conocimiento propio de $\mathrm{sí}^{41}$, aunque no equivalía al hábito de sabiduría. Polo, en cambio, sostiene que "el ser humano se alcanza con el hábito de sabiduría, que es un hábito innato"

Sellés sintetiza la explicación que hace Polo al innatismo de los hábitos referidos: "uno, porque dado que considera que el hombre es radicalmente bijo, en él todo es 'nato', es decir, que tales hábitos no son cognoscitivos desde el inicio de la existencia humana, sino que su conocer se activa en un momento determinado; otro, porque tales hábitos dependen en su activación del intelecto agente, por lo cual strictu senso no son innatos, sino que nacen de él. Por lo demás, se puede añadir que los tres son susceptibles de crecimiento, pero este desarrollo no acaece por repetición de actos, como el de algunos hábitos adquiridos de la razón o el de las virtudes de la voluntad, sino que obedece a la redundancia del acto de ser personal sobre ellos" ${ }^{\prime 3}$.

La concepción de Polo acerca de los hábitos innatos queda expuesta en varias de sus obras. Cada vez va existiendo más bibliografía secundaria acerca de estas cuestiones ${ }^{44}$.

sunt nata aliter nobis indita, ita in operativis sicut in speculativis. Et ideo sicut per principia praecognita facit aliquis inveniendo se scientem in actu". In Ethic., 1. II, lect. 4, n. 7.

38 "Habitus primorum principiorum non acquiritur per alias scientias, sed habet a natura". TOMÁS DE AQUINO, In I Sent., d. un., q. 1, a. 3, qc. b, ad 3; "in VI Ethicorum, inter alios habitus ponitur intellectus principiorum, qui est a natura, unde et principia prima dicunta nataaliter cognita". Summa Theologica, I-II, q. 51, a. 1, sc.

39 La sindéresis "est quodammodo innatus menti nostrae ex ipso lumine intellectus agentis, sicut et habitus principiorum speculativorum". TOMÁS DE AQUINO, In II Sent., d. 24, q. 2, a. 3 , co. "Praecepta legis naturalis quandoque considerantur in actu a ratione, quandoque autem sunt in ea habitualiter tantum, secundum nunc modum potest dici quod lex naturalis sit habitus. Sicut etiam principia indemonstrabilia in speculativis non sunt ipse habitus principiorum, sed sunt principia quorum est habitus”. Summa Theologica, I-II, q. 94, a. 1 co. Cfr. también: ibidem, ad 3.

40 "La sabiduría humana se tiene por adquisición". TOMÁS DE AQUINO, Super Ioh., VI, 1.

41 "Mens antequam a phantasmatibus abstrahat, sui notitiam habitualem habet, qua possit percipere se esse". TOMÁS DE AQUINO, De Veritate, q. 10, art. 8, ad 1.

42 L. POLO, Antropología I, 145.

43 J. F. SELLÉS, Los niveles cognoscitivos superiores de la persona humana, 55. Cfr. L. POLO, Curso de teoría IV, 679; El logos predicamental, 168; Nominalismo, Idealismo, Realismo, 261.

${ }^{44}$ Cfr. en torno al hábito de sabiduría: J. F. SELLÉS, "El hábito de sabiduría según Polo", en Studia Poliana, 3 (2001), 73-102. Sobre el hábito de los primeros principios: S. PIÁ TARAZONA, Los primeros principios en Leonardo Polo, Cuadernos de Anuario Filosófico, Serie de Filosofía Española, $\mathrm{n}^{\circ}$ 2, Servicio de Publicaciones de la Universidad de Navarra, Pamplona, 1997; S. FERNÁNDEZ, “'Intellectus principiorum': de Tomás de Aquino a Polo (y vuelta)”, en Anuario 
Una vez introducidos los hábitos innatos, su sentido, su correspondencia con la tradición filosófica medieval y aristotélica, es el momento de centrarnos en el primer abandono del límite mental.

De acuerdo con lo que se ha venido diciendo es preciso tener presente que el hábito de los primeros principios no pierde de vista la posesión inmanente del conocimiento objetivo, pero no lo posee aunque no lo pierda de vista. En otras palabras, "el hábito de los primeros principios equivale a una apertura hacia fuera según la cual se advierte el ser primero" ${ }^{35}$.

\section{CONOCIMIENTO HABITUAL DE LOS PRIMEROS PRINCIPIOS}

De modo preciso expone Polo lo que se propone con la primera dimensión del abandono del límite mental. "Como acto intelectual, la primera dimensión del abandono del límite es el hábito de los primeros principios. También cabe llamarla concentración atencional. Esta última expresión significa que el método sigue a los temas, es guiado por ellos sin dejar de iluminarlos, en tanto que como actos de ser no son estáticos: no son mismidades como los objetos. Por tanto, la concentración atencional se distingue de la operación cognoscitiva. La operación atiende según el objeto, que posee, pero no se puede llamar concentración atencional porque no tiene que plegarse a él, ya que el objeto es lo mismo. Por eso, la distinción entre la primera dimensión del abandono del límite y la operación es muy clara: en una se concentra la atención y en la otra no. La razón por la que se habla de primera dimensión del método reside en que, con ella, se declara la incompetencia de la operación para la advertencia del ser extramental”"

Filosófico, XXIX/2 (1996), 509-526; J. MORÁN, "Los primeros principios: interpretación de Polo de Aristóteles", en Anuario Filosófico, XXIX/2 (1996), 787-803; J. F. SELLÉS, "Sobre el sujeto y el hábito de los primeros principios. Una propuesta desde el 'corpus' tomista", en Miscelanea Poliana, 3 (2005), 20-29; "El carácter distintivo del hábito de los primeros principios", en Tópicos, 26 (2004), 153-176; "Unicidad e innatismo del hábito de los primeros principios. Un estudio desde el corpus tomista”, en Thémata, 34 (2005), 197-212. Sobre la sindéresis: F. MOLINA, "El yo y la sindéresis", en Studia Poliana, 3 (2001), 35-60; "Sindéresis y conciencia moral", en Anuario Filosófico, XXIX/2 (1996), 773-785; "Sindéresis y voluntad: ¿quién mueve a la voluntad?”, Futurizar el Presente, Universidad de Málaga, Málaga, 2003, 193-212; La sindéresis, Cuadernos de Anuario Filosófico, Serie Universitaria, n ${ }^{\circ}$, Servicio de Publicaciones de la Universidad de Navarra, Pamplona, 1999.

45 L. POLO, Antropología I, 191.

${ }^{46}$ L. POLO, Antropología I, 134. 
Para entender cabalmente lo que Polo quiere plantear en esta primera dimensión del abandono del límite mental conviene recordar, por un lado, la distinción aristotélica del acto -enérgeia y entelécheia-; por otro, el acto de ser de Tomás de Aquino que no es ni enérgeia ni entelécheia. Apoyándose en el acto de ser de Tomás de Aquino se abre un camino ascendente en la línea de los actos cognoscitivos. "Por asimilación al acto de ser, se puede hablar de actos cognoscitivos superiores a la enérgeia. Y si existe un acto superior a la enérgeia, la cual es un acto limitado (el conocimiento operativo es objetivo y por tanto limitado), cabe abandonar el límite. Abandonar el límite es encontrar otro sentido del acto intelectual que no es acto constitucional. Al ampliar la consideración del conocimiento más allá de la facultad, se aumenta su alcance temático" ${ }^{47}$.

Y así se puede plantear correctamente el tema del hábito intelectual, ya que decir que conocer habitualmente es conocer en acto no significa que el hábito sea la operación; a diferencia de la filosofía clásica para quien los principios son afrontados desde la objetividad.

¿Qué se conoce a través de los primeros principios? La respuesta a esta cuestión es nítida por parte de Polo: actus essendi. "Si los primeros principios son reales, y el hábito correspondiente tiene que ver con el actus essendi hominis, este hábito será el conocimiento del actus essendi (no de la entelécheia, sino de lo primordial como acto de ser)" ${ }^{48}$.

Por consiguiente, en una primera aproximación cabe señalar que el hábito de los primeros principios es el conocimiento en acto de los primeros principios, y los primeros principios son actos, son reales; por eso, el conocimiento de los primeros principios es el conocimiento del actus essendi: el desarrollo, el despliegue de la teoría del actus essendi de Tomás de Aquino.

Sin embargo, Polo hace notar enseguida la deficiencia objetiva que encuentra en la formulación de los primeros principios ${ }^{49}$. Para eso analiza las diversas aporías que encuentra en la formulación del Principio de no Contradicción, del Principio de Identidad y del Principio de Causalidad. Aporías que van a sugerir el abandono del objeto detectado como límite mental.

Sin embargo, al no ser las aporías una cuestión que aborde directamente la temática que estamos tratando, se remite al lector a aquellas páginas en las

\footnotetext{
${ }^{47}$ L. POLO, El conocimiento habitual de los primeros principios, 18.

${ }^{48}$ Ibidem, 19.

${ }^{49}$ Cfr. ibidem, 19-51.
} 
que lleva a cabo un pormenorizado estudio de esas aporías. Teniendo presente esas aporías, Polo afirma con nitidez que "existe un conocimiento habitual de los primeros principios y que el conocimiento de la no contradicción, y también de la identidad y de la causalidad en tanto que reales es habitual" ${ }^{50}$.

Dando un paso más acerca de qué conocen los primeros principios, hay que señalar que si el primer trascendental es el ser, los primeros principios se han de considerar como el conocimiento del ser en cuanto primero. "Por tanto, los primeros principios no pueden tener un valor meramente lógico y su conocimiento no es meramente objetivo" ${ }^{{ }_{1}}$.

De acuerdo con lo que venimos diciendo, podemos avanzar lo que Polo entiende por primeros principios. "Los primeros principios son la intelección insistente, concentrada, del acto de ser, o del ser en tanto que primero. De manera que con los primeros principios no abordamos directamente el tema de la verdad y del bien trascendentales, sino, en directo, el tema del ser trascendental como primero, como acto" ${ }^{\text {"2 }}$. Pero como no se trata de un solo principio, sino de tres, el conocimiento acerca del ser se amplía en la medida en que se intensifica ese conocimiento. Ahora bien, a esto hay que añadir un asunto que Polo considera muy importante: "se puede ser primero según la identidad, según la causalidad y según la no contradicción. Estas prioridades no se deben confundir" ${ }^{\prime 3}$.

Merece la pena indicar porqué este asunto es realmente importante para toda la filosofía que desarrolla Polo. Pienso que puede ayudar en la comprensión de lo que se viene diciendo la siguiente cita, aunque sea demasiado larga: "La orientación hacia este asunto, en mi caso, empezó cuando era muy joven. Tuve la suerte de encontrarme con un libro de Balmes, un filósofo español de la primera mitad del siglo XIX, que se llama Filosofía fundamental. Esta obra contiene una discusión con Descartes y con Kant. Balmes dice que es característico de la filosofía moderna no admitir más que un primer principio, y que eso es un error. Por tanto, la primordialidad del principio no es única. Naturalmente, en aquella edad tan sólo caí en la cuenta de que ahí había un problema muy serio y que debía desarrollar la sugerencia balmesiana en el supuesto de que me dedicara a la filosofía. En los comienzos de los años

50 Ibidem, 32.

${ }^{51}$ Ibidem, 52.

52 Ibidem, 53.

${ }^{53}$ Ibidem. 
cincuenta escribí unos papeles muy abundantes y muy tentativos, de los cuales entresaqué ese libro al que me he referido: El ser I. El ser I, en el fondo, es una refutación de la noción aristotélica de entelécheia y una manera de entender los tres primeros principios en tanto que distintos, pero vigentes entre sí, y como conocimiento del acto en sentido primordial, o acto de ser. Por eso suelo llamar a los primeros principios axiomas de la metafísica. Axiomas, porque los primeros principios se llaman axiomas; y de la metafísica, porque el tema central de la metafísica realista es el ser" ${ }^{\prime 4}$.

Dicho de otro modo, "los primeros principios no son manifestaciones o aspectos del ser, sino la intelección del ser en tanto que primero. Según la intelección, cabe ser como no contradicción, como causa y como identidad; o también: inteligir la no contradicción como ser, la causalidad como ser y la identidad como ser. Conseguir esto último es justamente la intelección de los primeros principios. Pero si los primeros principios son primeros como ser, hay que desechar su macla objetiva (hay que abandonar el límite mental)"55.

Dando un paso más dos cuestiones afloran: ¿qué significa conocer habitualmente?; dentro del conocimiento habitual -hay muchos hábitos intelectuales-, ¿qué es lo peculiar del hábito de los primeros principios?

\section{4. ¿QUÉ SIGNIFICA CONOCER HABITUALMENTE?}

Respecto a la primera cuestión, Polo se servirá de la cibernética para dar una explicación lo más nítida posible, pues tiene muy presente que el hombre es un ser cibernético. "La noción de hábito es de la más estricta actualidad histórica: es imposible aceptar que el hombre hace máquinas cibernéticas sin ser él cibernético" ${ }^{\circ 6}$. Aún más, "tiene que ser intrínsecamente cibernético. Y esto significa que el hombre es un sistema en el que la libertad incide" ${ }^{57}$.

Siguiendo a Tomás de Aquino, Polo señala un aspecto importante de ese conocer habitualmente consistente en señalar que los hábitos son el paso de la

\footnotetext{
${ }^{54}$ Ibidem, 53, nota 12.

55 L. POLO, El conocimiento habitual de los primeros principios, 68.

56 L. POLO, El conocimiento habitual de los primeros principios, 59. Para profundizar en esta cuestión, cfr. L. POLO, "La cibernética como lógica de la vida", en Studia Poliana, 4 (2002), 9-17. Otros autores que han tratado esta misma cuestión: F. HAYA, Tomás de Aquino frente a la crítica: La articulación trascendental de conocimiento y ser, Eunsa, Pamplona, 1992; J. I. MURILLO, El conocimiento como ser, Eunsa, Pamplona, 1993; J. F. SELLÉS, Conocer y amar, Eunsa, Pamplona, 1995; J. M. POSADA, Una física de causas, Eunsa, Pamplona, 1996.

57 L. POLO, El conocimiento habitual de los primeros principios, 59.
} 
libertad a la naturaleza. "Los hábitos no sólo cumplen la función de modificar un sistema en virtud de una realimentación, de transformar la salida en entrada al captar el valor significativo de la salida (consideración del autocontrol de un sistema por realimentación); los hábitos no solamente perfeccionan la facultad (lo cual es común a la inteligencia y a la voluntad), sino que son el paso de la libertad a la naturaleza: sin hábitos, una naturaleza no es libre" ${ }^{158}$.

Por eso no es adecuado decir que la libertad es una propiedad de la voluntad, sino que más bien la libertad la alcanza en la medida en que es capaz de hábitos. Sin hábitos la voluntad es pura potencia pasiva en orden al fin (voluntas ut natura). Y lo mismo pasa con la inteligencia, pues los hábitos intelectuales conectan la libertad con la inteligencia.

Con este planteamiento, la libertad no radica en las facultades, por lo que hay que situarla en el esse hominis. "No está en el orden de la natura, sino que puede tomar contacto con ella en tanto que existen hábitos. Sin hábitos, la natura está cerrada a la libertad, lo cual no es una impotencia de la libertad, sino una incapacidad de la natura. Si existe la libertad, el absoluto no es el resultado, porque la libertad es incompatible con la idea de todo" ${ }^{59}$.

Hemos visto, someramente, lo que significa conocer habitualmente. Antes de entrar en la segunda cuestión planteada, conviene señalar que los hábitos intelectuales son el conocimiento de la operación que permite el perfeccionamiento de la inteligencia. "Un hábito intelectual no es simplemente el perfeccionamiento de la inteligencia por haber ejercido una operación, sino que el hábito intelectual es el conocimiento de la operación" ${ }^{60}$. Por tanto, la respuesta a la cuestión sobre qué significa conocer habitualmente debiera ser, conocer la operación. Y esto porque la operación lo que conoce es al objeto, pero no a sí misma.

De acuerdo con lo que se viene señalando, sin hábito, no podríamos conocer la enérgeia, porque la enérgeia no se puede conocer a sí misma. De manera que para formular la noción de enérgeia, para decir que el conocimiento es acto como operación, es menester que se conozca la operación. "La enérgeia se caracteriza porque conoce un objeto sin formar parte de ese objeto, sin constituirlo ni conocerse en él: es un acto intelectual, no una acción productiva; el objeto es intencional, no real”' ${ }^{\prime 1}$.

58 Ibidem.

59 Ibidem.

${ }^{60}$ Ibidem, 60.

${ }^{61}$ Ibidem, 61. 
Polo insistirá en que ninguna operación cognoscitiva se conoce a sí misma. La presencia del hábito es necesaria para que se pueda dar el conocimiento de la operación cognoscitiva. Lo formula de otro modo: "si la operación no se puede conocer como objeto, y, por otra parte, las operaciones son conocidas, es menester otro acto cognoscitivo más alto" ${ }^{62}$. Y así la operación ejercida es la temática de cada uno de los hábitos, de modo que el hábito es ante todo el conocimiento de la operación intelectual.

\section{5. ¿QUÉ ES LO PECULIAR DEL HÁBITO DE LOS PRIMEROS PRINCIPIOS?}

Veamos ahora la segunda cuestión que nos quedó pendiente: ¿qué es lo peculiar del hábito de los primeros principios?

Como ya se advirtiera, sin ser el hábito más importante el de los primeros principios -recordemos que la sabiduría es más alto-, su conocimiento versa sobre el acto de ser. De este modo, "existe un hábito peculiar que, al conocer el carácter limitado de la operación, conoce prioridades superiores a la operación. Esas prioridades superiores a la enérgeia son los primeros principios, y ese hábito es el intellectus ut babitus" ${ }^{\prime 3}$. El tema de este hábito es, por consiguiente, la aprioridad del acto de ser. Ahora bien, esa aprioridad no quiere decir que conozca el ser personal.

Lo propio, por tanto, en el hábito de los primeros principios más que iluminar la enérgeia lo que se ilumina es que la operación cognoscitiva es límite y que existen prioridades superiores a ella. Esas prioridades superiores son los primeros principios, conocidos habitualmente. Así es como se conoce, de acuerdo con Polo, el actus essendi. Puede decirse entonces que "el abandono de la presencia mental como operación es el hábito de los primeros principios" ${ }^{4}$.

Los primeros principios son pues la intelección del ser en tanto que primero. Y según sea la intelección del ser, "cabe ser como no contradicción, como causa y como identidad; o también: inteligir la no contradicción como ser, la causalidad como ser y la identidad como ser. Conseguir esto último es justamente la intelección de los primeros principios" ${ }^{55}$.

${ }^{62}$ Ibidem.

${ }^{63}$ Ibidem, 63.

${ }^{64}$ L. POLO, Curso de teoría del conocimiento IV/I, 39, nota 25.

${ }^{65}$ L. POLO, El conocimiento habitual de los primeros principios, 68-69. El estudio de las maclas -inclusión de un principio en el otro- entre los primeros principios, por lo que Polo entiende confusión y no ser capaz de establecer una prioridad y una distinción clara en el modo de llegar al 


\section{6. ¿QUÚ CONOCIMIENTO DE Dios SE ALCANZA EN LA PRIMERA DIMENSIÓN DEL ABANDONO DEL LÍMITE MENTAL?}

Dentro de los primeros principios el más alto de ellos es el principio de identidad que es el conocimiento de Dios. "Dios se conoce como el primer principio que le corresponde ser a Él Mismo. Precisamente por eso, lo peculiar del conocimiento de la identidad es su carácter originario. El carácter originario se hurta a la actualidad objetiva. Ello no impide el conocer en presente, pero en ese nivel se pierde el esse divino. El principio de identidad es temático en tanto que se conoce habitualmente"

Y su temática radica en la intelección del acto de ser increado. "El acto de ser de la criatura se distingue del acto de ser de Dios no porque sea distinto realmente de la esencia, sino por ser creado. Si esto no se tiene en cuenta, se cae en un indiscernimiento unilateral del acto de ser. Por consiguiente, hay que aceptar la distinción entre Dios y la criatura en la línea del acto de ser, la cual, a su vez, comporta en la criatura distinción del ser con la esencia" ${ }^{\prime 7}$.

A través de los primeros principios, detectamos el límite de la operación cognoscitiva que nos lleva a su abandono y el conocimiento que se alcanza de Dios consiste en que "podemos llegar habitualmente a que Dios es Origen y el Origen es el estricto significado de la Identidad, de tal manera que la Identidad es Originaria. Si en la Identidad hubiese algo además del Origen, alguna continuación, algún llegar a ser idéntico, no sería primer principio" ${ }^{8}$.

acto de ser o ser primero de modo diverso, puede verse con más profundidad en las siguientes obras: Teoría del Conocimiento IV/I, El conocimiento babitual de los primeros principios, Antropología I, El Ser, Acceso al ser. Es un tema que Polo tiene muy presente pues si se puede resolver esa macla -no resuelta por los clásicos y modernos- entonces es factible detectar el límite y estar en condiciones de abandonarlo.

${ }^{66}$ L. POLO, Curso de teoría del conocimiento IV/I, 48.

${ }^{67}$ L. POLO, Antropología I, 117. Cfr. Persona y libertad, 39-40.

${ }^{68} \mathrm{~L}$. POLO, El conocimiento habitual de los primeros principios, 72. 